\title{
Thermodynamic considerations of same- metal electrodes in an asymmetric cell
}

\author{
M. H. Braga ${ }^{1,2^{*}}$ (D, N. S. Grundish ${ }^{1}$, A. J. Murchison ${ }^{1}$ and J. B. Goodenough ${ }^{1 *}$
}

\footnotetext{
* Correspondence: mbraga@fe.up. pt; jgoodenough@mail.utexas.edu ${ }^{1}$ Texas Materials Institute and the Materials Science and Engineering Program, The University of Texas at Austin, Austin, TX, USA Full list of author information is available at the end of the article
}

\begin{abstract}
An electrochemical cell contains three open thermodynamic systems that, in dynamic equilibrium, equalize their electrochemical potentials with that of their surrounding by forming an electric-double-layer-capacitor at the interface of the electrolyte with each of the two electrodes. Since the electrode/electrolyte interfaces are heterojunctions, the electrochemical potentials or Fermi levels of the two materials that contact the electrolyte at the two electrodes determine the voltage of a cell. The voltage is the sum of the voltages of the two interfacial electric-double-layer capacitors at the two electrode/electrolyte interfaces. A theoretical analysis of the thermodynamics that gives a quantitative prediction of the observed voltages in an asymmetric cell with an $\mathrm{S}_{8}$ relay at the positive electrode is provided. In addition, new discharge data and an X-ray photoelectron spectroscopy analysis of the lithium plated on the positive electrode of a discharged cell is presented. Ab initio, DFT methods were used to calculate the band structure and surface-state energies of the crystalline $S_{8}$ solid sulfur relay. The theoretical exposition of the thermodynamics of the operative driving force of the chemical reactions in an electrochemical cell demonstrate that our initial experimental data and conclusions are valid. Other reported observations of lithium plating on the positive electrode, observations that were neither exploited nor their origins specified, are also cited.
\end{abstract}

Keywords: Thermodynamics, Electrochemical potentials, Heterojunctions, Batteries, Solid state, Double layer capacitors

\section{Introduction}

In order to clarify that our observations (Braga et al. 2017) of the transfer on discharge of a lithium anode to the cathode conforms to the laws of thermodynamics, the First Law of Thermodynamics for a closed and an open system is rehearsed (The first law of thermodynamics); the electrode/electrolyte interfaces introduce applied forces that perform work on the mobile charges of a cell. Therefore, although a battery cell is a closed system, it contains three open systems, the two electrodes and the electrolyte. Our thermodynamic analysis provides quantitative predictions of our observed voltages for a cell with a sulfur relay. Moreover, it is illustrated how the electric-double-layer capacitors (EDLCs) are formed at the electrode/electrolyte interfaces in order to equalize the electrochemical potentials of the two materials at an interface. In Section "Experimental", additional experimental data corroborating plating on the positive electrode of an asymmetric cell in a discharge at a finite voltage is provided. In addition, it is shown how the electrochemical potentials of the phases

(C) The Author(s). 2019 Open Access This article is distributed under the terms of the Creative Commons Attribution 4.0 International License (http://creativecommons.org/licenses/by/4.0/), which permits unrestricted use, distribution, and reproduction in any medium, provided you give appropriate credit to the original author(s) and the source, provide a link to the Creative Commons license, and indicate if changes were made. 
and elements in each phase vary in a mixture depending of the surrounding contacting-phase. Examples from the literature of plating of an alkali-metal negative electrode on the positive electrode at a finite voltage are cited, observations that were neither utilized nor fully understood.

\section{The first law of thermodynamics}

A closed system is a physical system that does not exchange matter with the surroundings but it exchanges energy. An isolated system cannot exchange any heat, work, or matter with the surroundings, but an open system can exchange energy and matter (Prigogine and Defay 1950/1954). The first law of Thermodynamics states that in a closed system,

$$
d U=\delta Q-\partial W
$$

where $d U$ is the change in internal energy, $\delta Q$ is the heat added to the system, and $\partial W$ is the work made by the system.

In an open system, the internal energy $d U$ includes changes in the chemical potential made by applied forces. The applied force may, for example, be an electric, magnetic or a gravitic force to make

$$
d U=T d S-P d \tilde{V}+\sum_{i} \mu_{i} d N_{i^{-}} \sum_{i} X_{i} d a_{i}
$$

where $P$ is the pressure and $\tilde{V}$ the volume of the system, $\mu_{i}$ is the chemical potential of particles $i$ of the system, $d N_{i}$ is the infinitesimal increase in particles $i$ of the system (if $d N_{i}$ is expressed in mol then $\mu_{i}$ is expressed in $\mathrm{J}^{\mathrm{mol}}{ }^{-1}$ ), $X_{i}$ is an external generalized force, an intrinsic property, while $a_{i}$ is an extrinsic property (dependent on the size). The term $-P d \tilde{V}$ can be included in $-\sum_{i} X_{i} d a_{i}$. For a gravitic force, $-\sum_{i} X_{i} d a_{i}$ $=-\sum_{i} m_{i} \boldsymbol{g} \cdot d \boldsymbol{r}_{i}=-\partial W_{g}$ where $m_{i}$ is the mass of particle $i, \boldsymbol{g}$ is the gravitic acceleration, and $d \boldsymbol{r}_{i}$ a distance in which gravitic work, $W_{g}$, was performed. In the case of electric forces, $-\sum_{i} X_{i} d a_{i}=-\sum_{i} q_{i} E . d \boldsymbol{r}_{i}=-\partial W_{e}$ is the electrical work done on particles $i$ of the system containing charge $q_{i}$ by an external electric force $q_{i} \boldsymbol{E} ; \boldsymbol{E}$ is the electric field, which hypothetically moves $q_{i}$ a distance $d \boldsymbol{r}_{i}$, and $\boldsymbol{E} . d \boldsymbol{r}_{i}=-d V_{i}$ where $V_{i}$ is the electric potential to which particles $i$ are subjected. The Gibbs free energy is,

$$
G=H-T S
$$

where $H$ is the enthalpy, $S$ the entropy, and $T$ the temperature in $\mathrm{K}$. The enthalpy is,

$$
H=U+P \tilde{V}
$$

Therefore,

$$
\mathrm{d} G=\mathrm{d} U+P d \tilde{V}+\tilde{V} d P-T \mathrm{~d} S-S \mathrm{~d} T
$$

and from (2), 


$$
d G=\tilde{V} d P-S \mathrm{~d} T+\sum_{i} \mu_{i} d N_{i}+\sum_{i} q_{i} d V_{i}
$$

For a constant $P$ and $T$, Eq. (6) becomes,

$$
d G=\sum_{i} \mu_{i} d N_{i}+\sum_{i} q_{i} d V_{i}
$$

The electrochemical potential of an open system of particles $i$ or species $i$ is,

$$
\bar{\mu}_{i}=\left(\frac{\partial G}{\partial N_{i}}\right)_{T, P, N_{j \neq i}}
$$

From (7) and (8),

$$
\bar{\mu}_{i}=\mu_{i}+z_{i} F \phi_{i}
$$

where $\bar{\mu}_{i}$ is the electrochemical potential of particles $i$ in J.mol ${ }^{-1}$ and $\mu_{i}$ is the chemical potential of particles $i$ of valence $z_{i}$ in $\mathrm{J}^{\mathrm{mol}}{ }^{-1} ; F$ is the Faraday constant in C.mol ${ }^{-1}$; and $\phi_{i}$ is the local electrostatic potential in V (Trasatti 1986; Bardeen 1947). Equation (9) is fundamental to explain how a battery cell works. Furthermore, it explains how other electrical devices like transistors (Bardeen 1947), electrochromic devices (Heo et al. 2017), diodes (Sparkes 1994), light emitting diodes (LED) (Schubert 2006), thermionic diodes (Jenkins and Jarvis 2013), photovoltaic cells (NREL 1982), capacitors (Kaiser 2011) and kelvin probes (Nonnenmacher et al. 1991) work as a result of the equalization of the electrochemical potentials at the interfaces of two materials in contact with one another.

A system and its surroundings can jointly be considered a closed system at constant $P$ and $\tilde{V}$. As a closed system, they will achieve a steady-state (thermodynamic dynamic equilibrium) when the Gibbs free energy is minimum, which is when $d G=0$,

$$
d G=0 \Rightarrow \sum_{i} \mu_{i} d N_{i}=-\sum_{i} q_{i} d V_{i}
$$

Although a battery cell can be considered a closed system, it contains three open systems (the negative and positive electrodes and the electrolyte) to which an external electric field from the material contacted is applied.

On the other hand, according to Gauss's law for ideal charged parallel plates (electrode/electrolyte interface), the electric field outside the internal space between plates is zero, i.e., the electric field outside the double-layer-capacitor (EDLC) is zero; therefore, the electric field to which the electrodes are subjected is the electric field at the interface with the electrolyte (the magnitude of the electric field inside an electrode/ electrolyte EDLC can be of the order of $10^{8} \mathrm{Vm}^{-1}$ ).

Since the whole battery cell is a closed system (the heat exchanges with the surroundings due to the Joule effect are not considered since they depend on the internal resistance, which varies from cell to cell), Eq. (10) applies if the chemical potentials are taken relative to an internal reference. If the chemical potential of the electrolyte is taken as the reference (Fig. 1), Eq. (11) follows, 


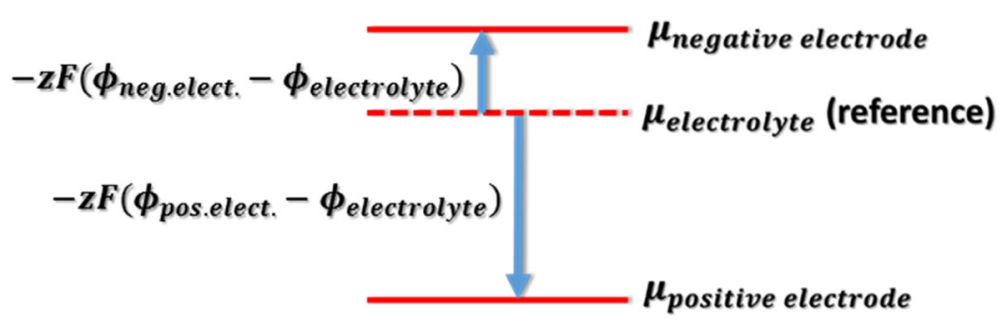

Fig. 1 Schematics of the chemical potentials in a closed system battery cell when the electrolyte chemical potential is the reference

$$
\begin{aligned}
& \bar{\mu}_{\text {pos.elect. }}-\bar{\mu}_{\text {neg.elect }}=0 \Rightarrow\left(\mu_{\text {neg.elect. }}-\mu_{\text {electrolyte }}\right)-\left(\mu_{\text {pos.elect. }}-\mu_{\text {electrolyte }}\right) \\
& =-z F\left(\phi_{\text {neg.elect. }}-\phi_{\text {electrolyte }}\right)+z F\left(\phi_{\text {pos.elect. }}-\phi_{\text {electrolyte }}\right)= \\
& =-z F\left(\Delta \phi_{E D L C\left(\frac{\text { neg.elect }}{\text { electroble }}\right)}-\Delta \phi_{E D L C\left(\frac{\text { poselect }}{\text { electroblec }}\right)}\right)=-z F \Delta V \Leftrightarrow \bar{\mu}_{\text {pos.elect. }}-\bar{\mu}_{\text {neg. elect }} \\
& =0 \Leftrightarrow \mu_{\text {neg.elect. }}-\mu_{\text {pos.elect. }}=-z F \Delta V
\end{aligned}
$$

where $\Delta V$ is the potential difference between the two electrodes of a battery cell.

If the positive electrode is the reference,

$$
\begin{aligned}
& \bar{\mu}_{\text {neg.elect. }}-\bar{\mu}_{\text {electrolyte }}=0 \Rightarrow\left(\mu_{\text {neg.elect. }}-\mu_{\text {pos. elect. }}\right)-\left(\mu_{\text {electrolyte }}-\mu_{\text {pos.elect. }}\right)= \\
& =-z F\left(\phi_{\text {neg.elect. }}-\phi_{\text {electrolyte }}\right)=-z F \Delta \phi_{E D L C\left(\frac{\text { negeclect. }}{(\text { electrolyee }}\right)} \bar{\mu}_{\text {neg. elect. }}-\bar{\mu}_{\text {electrolyte }} \\
& =0 \Leftrightarrow \mu_{\text {neg. } \text {.lect. }}-\mu_{\text {electrolyte }}=-z F \Delta \phi_{E D L C\left(\frac{\text { negerect }}{\text { electroble }}\right)}
\end{aligned}
$$

Finally, with the negative electrode as reference,

$$
\begin{aligned}
& \bar{\mu}_{\text {pos.elect. }}-\bar{\mu}_{\text {electrolyte }}=0 \Rightarrow\left(\mu_{\text {pos.elect. }}-\mu_{\text {neg.elect. }}\right)-\left(\mu_{\text {electrolyte }}-\mu_{\text {neg.elect. }}\right)= \\
& =-z F\left(\phi_{\text {pos.elect. }}-\phi_{\text {electrolyte }}\right)=-z F \Delta \phi_{E D L C\left(\frac{\text { poselect }}{\text { dectroble })}\right)} \bar{\mu}_{\text {pos.elect. }}-\bar{\mu}_{\text {electrolyte }} \\
& =0 \Leftrightarrow \mu_{\text {pos.elect. }}-\mu_{\text {electrolyte }}=-z F \Delta \phi_{E D L C\left(\frac{\text { poselect }}{\text { electroblec }}\right)}
\end{aligned}
$$

Equations (11) to (13) indicate that in a battery cell, not only are the electrodes in dynamic equilibrium, but also each electrode with the electrolyte. Equation (11) is commonly used to relate the electrochemical potentials of the electrodes with a cell's potential at, any time in a dynamic equilibrium (steady-state)

$$
\mu_{\text {neg.elect. }}-\mu_{\text {pos.elect. }}=\Delta G=-w_{e}=-z F \Delta V
$$

or at open circuit prior to charge or discharge,

$$
\mu_{\text {neg.elect. }}^{0}-\mu_{\text {pos.elect. }}^{0}=\Delta G^{\circ}=-z F \Delta V^{\circ}
$$

During the reaction occurring in a battery cell at closed circuit,

$$
\Delta G=\Delta G^{\circ}+R T \ln \left(Q_{r}\right)
$$

where $\Delta G$ is the Gibbs free energy change per mole of reaction, $w_{e}$ is the electrical 
work per mole of reaction, $\Delta G^{\circ}$ is the Gibbs free energy change per mole of reaction for unmixed reactants and products at standard conditions, $\Delta V^{\circ}$ standard cell potential, $Q_{r}=e^{-\frac{2 F\left(\Delta V-\Delta V^{\circ}\right)}{R T}}$ is the reaction coefficient.

At equilibrium (at open circuit),

$$
\Delta G^{\circ}=-R T \ln \left(K_{e}\right)
$$

where $K_{e}$ is the equilibrium constant.

$$
K_{e}=e^{-\frac{\Delta G^{\circ}}{R T}}=e^{+\frac{z F \Delta V^{\circ}}{R T}}
$$

\section{Thermodynamics in an all-solid state Li-S cell}

The universal law that states that two dissimilar solid materials in contact equalize their electrochemical potential (or Fermi level) $\bar{\mu}_{i}$ applies not only separately to each electrode/electrolyte interface, but also between the two electrodes in contact across the electrolyte. Therefore, in a battery containing a negative electrode (e.g. lithium, $z_{i}$ $=1$ ) and a positive electrode (e.g. sulfur, $S_{8}$ ) each electrode equalizes its electrochemical potential with the electrochemical potential of the electrolyte by the formation of an EDLC at each electrolyte interface through the movement of ions in the electrolyte, see Fig. 2. For this system, Eq. (11) becomes,

$$
\bar{\mu}_{L i}-\bar{\mu}_{S_{8}}=0=\mu_{L i}-\mu_{S_{8}}+F\left(\phi_{L i}-\phi_{S_{8}}\right) \Rightarrow \mu_{L i}-\mu_{S_{8}}=-F\left(\phi_{L i}-\phi_{S_{8}}\right)=-F \Delta V
$$

where $\Delta V$ is the voltage of the cell at any given time.

From (11) and (19) the difference in chemical potentials in a Li-S battery cell is,

$$
\mu_{L i}-\mu_{S_{8}}=-F\left(\Delta \phi_{E D L C\left(\frac{L i}{\text { electroble }}\right)}-\Delta \phi_{E D L C\left(\frac{s_{8}}{\text { electrolyte }}\right)}\right)=-F \Delta V
$$

It is emphasized that two dissimilar solid materials in contact equalize their electrochemical potentials (Fermi levels); therefore, the alignment of the Fermi levels happens in each electrode independent of the rest of the cell. Conductors in contact align their electrochemical potentials by forming EDLCs with/without tunneling of electrons. In the positive electrode, the $\mathrm{S}_{8}$ insulator in contact with the electrolyte determines the electrochemical potential of the positive electrode Fig. 2b. While the empty surface states (Bardeen 1947) of the relay insulator face the electrolyte, the filled surface states on the opposite surface repel the incoming electrons from the negative electrode Figs. 2, 3 and 4. It is also highlighted that the band gaps are usually underestimated in DFT calculations (Brothers et al. 2008) by a factor of ca. 1.6. The band gap of $\mathrm{S}_{8}(\mathrm{~s})$ in Fig. $3 \mathrm{~b}$ can be as wide as $\sim 3.2 \mathrm{eV}$ and the Mott-Hubbard gap of the surface states is $\sim 0.4 \mathrm{eV}$ (see Additional file 1).

Rivest et al. (Rivest et al. 2014) explored the possibility to tune the electrochemical potential of $\mathrm{TiO}_{2}$ in gas and liquid environments by controlled attachment of relay phosphonic acid absorbates, organic insulator molecules containing dipoles. This "controlled attachment" formed EDLCs at the surface that provided the necessary energy to set the electrochemical potential of the $\mathrm{TiO}_{2}$.

In our cells in (Braga et al. 2017), the plated lithium in contact with the positive electrode equalizes its electrochemical potential with that of the carbon, which is 

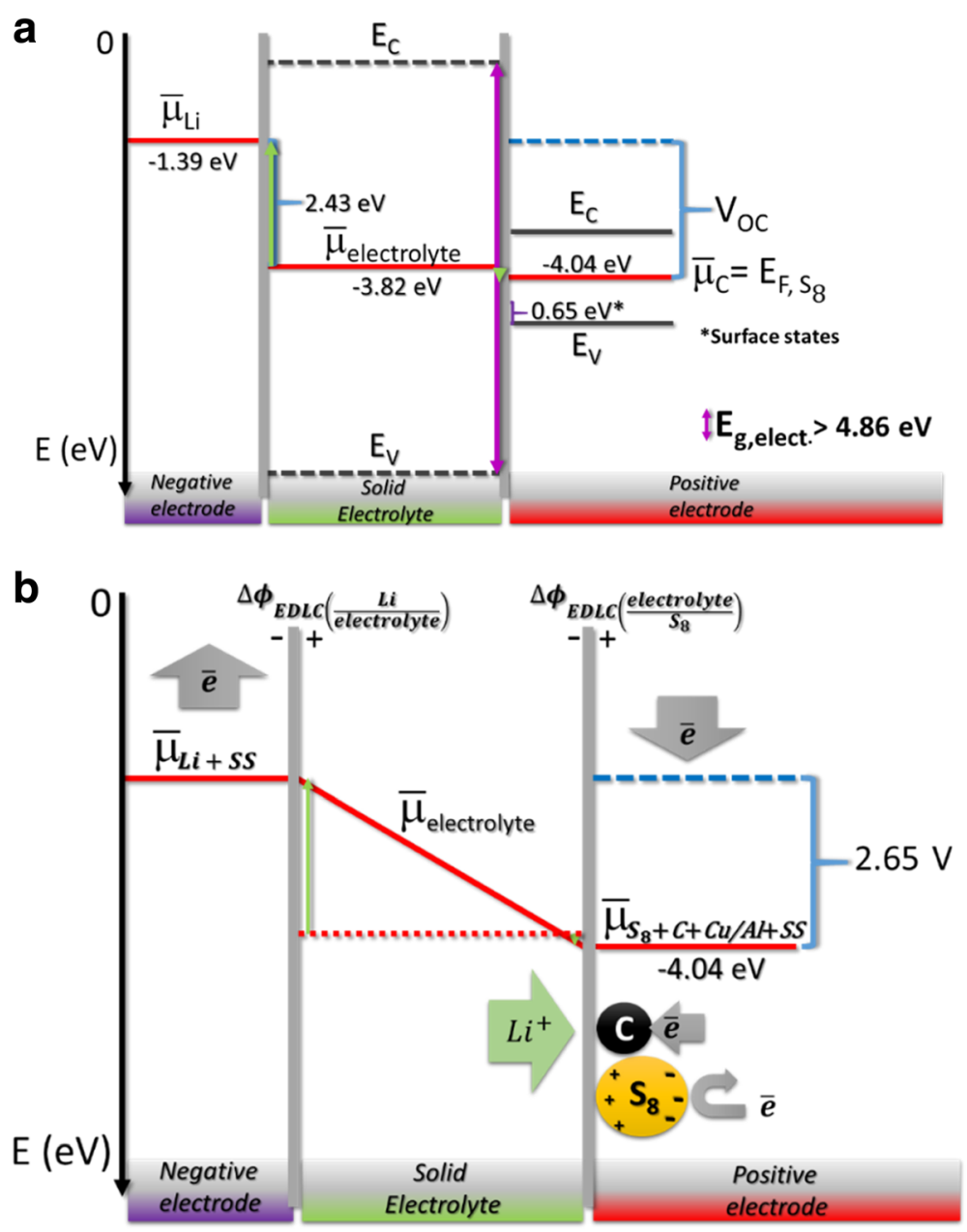

Fig. 2 Li-S all-solid state battery cell containing stainless steel (SS) current collectors in contact with both electrodes, lithium negative electrode and sulfur $\mathrm{S}_{8}+$ carbon positive-electrode with copper or aluminum positive current collector; $\mathbf{a}$ before equalization of the electrochemical potentials of the electrodes with the electrolyte; $\mathbf{b}$ after equalization of the electrochemical potentials during discharge. The electrochemical potential of the positive electrode is smaller than the chemical potential of the unmixed electrolyte reference. The energy loss by the Joule effect due to the resistances inside the cell was not considered.

equalized with the electrochemical potential of the $\mathrm{S}_{8}$. Therefore, the electrochemical potential of the lithium plated on the positive electrode is not equal to the chemical potential of the negative-electrode lithium, $\bar{\mu}_{\text {Li@positive electrode }} \neq \bar{\mu}_{L i @ \text { negative electrode, }}$, it is $\bar{\mu}_{L i}=\bar{\mu}_{S_{8}}$ as in Eqs. (19) and (20). The carbon and copper help to equalize the Fermi level of the lithium with the sulfur since these metals have to equalize their Fermi levels by receiving electrons from the lithium:

$$
\mu_{L i}-\mu_{L i @ S_{8}} \text { pos.elec }=-F\left(\Delta \phi_{E D L C_{\text {electrobte }}}-\Delta \phi_{E D L C \frac{L i S_{8}}{\text { electrostyle }}}\right)=-F \Delta V
$$

The Gibbs energy of the lithium plating reaction at a sulfur positive electrode from a lithium negative electrode is the same as the Gibbs energy available in a traditional Li-S cell before sulfur reduction (Fig. 2b) provided the lithium does not cover the sulfur in contact with the electrolyte. 


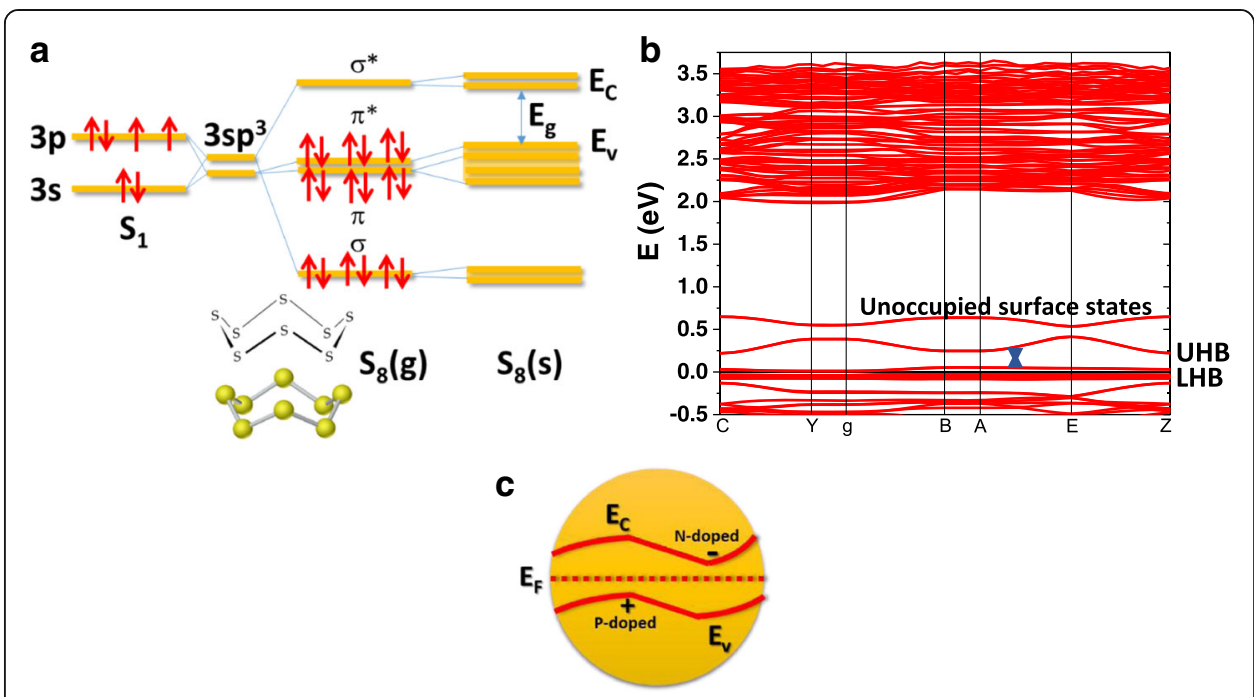

Fig. 3 Sulfur electronic structure; a electronic structure for an $\mathrm{S}$ atom, $\mathrm{sp}^{3}$ hybridization, bonding and nonbonding molecular orbitals for $\mathrm{S}_{8}(\mathrm{~g})$ and band structure for $\mathrm{S}_{8}(\mathrm{~s}) \mathbf{b}$ first principles simulated band structure for monoclinic $\mathrm{P} 2 / \mathrm{C}_{8}(\mathrm{~s})$, containing 128 atoms and a simulated surface (where periodicity is broken). $\mathrm{E}_{\mathrm{c}}$ is bottom conduction band, $E_{v}$ is top valence band, UHB is upper Hubbard band, LHB is lower Hubbard band highlighting the Mott-Hubbard gap between filled and empty states of the surface's dangling bonds; c Schematic representation of an $\mathrm{S}_{8}$ (s) particle showing band bending (Bardeen 1947) at the surface allowing the Fermi level to be the same across the particle and forming a dipole (sulfur dielectric constant is 1.6-1.7)

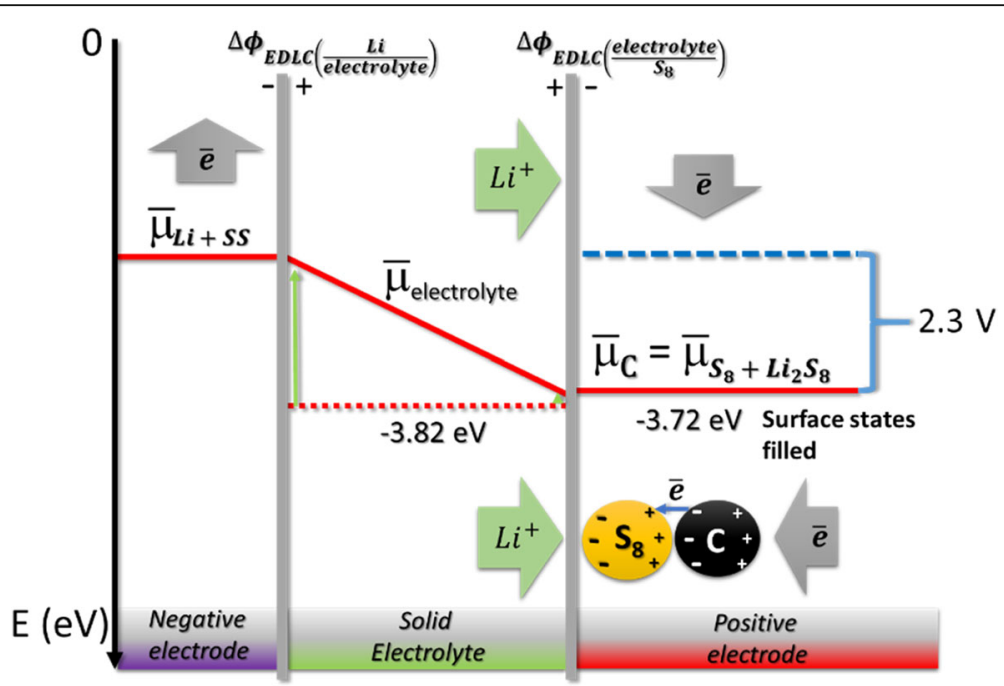

Fig. 4 During discharge of a Li-S all-solid state battery cell containing stainless steel current collectors in contact with both electrodes, lithium negative electrode, sulfur $S_{8}$ and carbon positive electrode and copper or aluminum positive current collector. If the electrochemical potential of the positive electrode increases until it overcomes $-3.82 \mathrm{eV}$, the EDLC charges flip in order to equalize the electrochemical potentials and more Li-ions are attracted to the electrolyte interface with the positive electrode. We do not consider the energy lost by the Joule effect due to the resistances inside the cell 
During discharge, the Fermi level of the sulfur remains in the $\mathrm{S}_{8}$ energy gap so long as the rate of the arrival of electrons from the negative electrode is lower than the rate of tunnelling of electrons to surface $\mathrm{Li}^{+}$to plate lithium $\mathrm{Li}^{0}$ on the conductive components of the positive electrodes. However, at faster rates of discharge, the electrons may fill the empty surface states of the relay and begin to reduce the relay, which makes the relay act like a conventional host cathode particle.

As shown in Eq. (2), if two locations have different total chemical potentials for a certain species, some of it may be due to potentials associated with "external" force fields (electric potential energy differences, gravitational potential energy differences, etc.), while the rest may be due to "internal" factors (density, temperature, etc.) (Kittel and Kroemer 1980). Therefore, the total chemical potential can be split into internal chemical potential and external chemical potential (Kittel and Kroemer 1980),

$$
\mu_{\text {total }}=\mu_{\text {int }}+\mu_{\text {ext }}
$$

where

$$
\mu_{\text {ext }}=q V+m g h+\ldots
$$

i.e., the external potential is the sum of electric potential, gravitational potential, etc. ( $q$ and $m$ are the charge and mass of the species, respectively, $V$ and $h$ are the voltage and height of the container, respectively, and $g$ is the acceleration due to gravity.) The internal chemical potential (usually just referred to as chemical potential) includes everything else besides the external potentials, such as density, temperature, and enthalpy.

In equilibrium, it follows

$$
\Delta \mu_{\text {int }}=-\Delta \mu_{\text {ext }}
$$

which is similar to (10) when the external work is just electrical. C. Kittel and H. Kroemer state (Kittel and Kroemer 1980): "The chemical potential is equivalent to a true energy: the difference in chemical potential between two systems is equal to the potential barrier that will bring the two systems to diffusive equilibrium".

During discharge, as is shown in Fig. 2, the sulfur repels the positive electrode's incoming electrons from the negative electrode until the electrochemical potential of the positive electrode reaches $-3.82 \mathrm{eV}$, which is the chemical potential of the electrolyte; at this positive-electrode electrochemical potential, the EDLC at the positive electrode and electrolyte interface ceases to exist. In Fig. 3, the positive electrode's electrochemical potential is $-3.72 \mathrm{eV}$; at this voltage, the surface states are filled and the electrochemical potential of the positive electrode is higher than the electrolyte's chemical potential; therefore, the EDLC at the positive electrode's interface is reversed and the $S_{8}$ is reduced.

In the conditions of Fig. 2, $\Delta \phi_{E D L C\left(\frac{\text { electrobyte }}{S_{8}}\right)}=0.22 \mathrm{~V}$ and an electron at the interface has a probability of $38 \%$ (see SI) to tunnel through the triangular quantum barrier. Upon electron tunneling a $\mathrm{Li}^{+}$at the surface of the electrolyte is reduced to $\mathrm{Li}^{0} ; \mathrm{Li}^{0}$ diffuses toward the positive electrode according to Fick's law of diffusion. The diffusion of $\mathrm{Li}^{0}$ is easier than the diffusion of the $\mathrm{Li}$-ion, since $\mathrm{Li}^{+}$are trapped at the EDLC by coulombic forces. The lithium at the surface of the carbon equalizes its Fermi level with that of the positive electrode by giving up some of its electrons and becoming positively charged. When the cell's potential is $2.5 \mathrm{~V}$, the triangular energy barrier at 
the electrolyte/positive electrode surface EDLC becomes $0.07 \mathrm{eV}$; the latter energy is found assuming that the Fermi level of the negative electrode does not vary much since lithium is a sink of electrons containing many more free electrons than those aligned at the surface forming the EDLC with the electrolyte. The probability of an electron to be transmitted through the triangular quantum barrier of $0.07 \mathrm{eV}$ at the EDLC, increases to $58 \%$ for a barrier width $d=0.3 \mathrm{~nm}$ (EDLCs thicknesses vary between $0.3-0.8 \mathrm{~nm}$ (Bardeen 1947)). Here the energy lost by the Joule effect due to the resistance to the movement of the ions and electrons across the electrolyte and electrodes was not accounted for since the battery cell was considered a closed system. So long as the electrochemical potential of the positive electrode exceeds that of the electrolyte, electrons tunnel through a potential well to adsorbed $\mathrm{Li}^{+}$as shown in Fig. 4.

In the cells in (Braga et al. 2017), the solid electrolyte contains dipoles. The dipoles help to keep the charges aligned close to the EDLCs at the interfaces according to the schematics in Fig. 5. Moreover, additional phenomena will take place due to the long-term alignment of dipoles.

\section{Electrochemical potentials in mixtures}

In a battery cell, the reactions occurring at negative and positive electrodes result in a change of electrochemical potential as shown in Eqs. (14) to (18). In an electrode, as in other mixtures of material phases, a two-phase dynamic equilibrium translates into the equalization of electrochemical potentials of the two phases in contact.

Here the Li-Si system was used to illustrate what can happen at the electrodes of a battery cell. In Fig. 6 two graphs from (Liang et al. 2017) show how the electrochemical potentials of the stoichiometric phases of the Li-Si system vary at constant temperature. A stoichiometric phase step-varies its electrochemical potential as it contacts with different phases as shown in Fig. 6a).

Figure 7 shows how the electrochemical potential of the lithium versus liquid-lithium varies (Liang et al. 2017; Braga et al. 1995; Braga et al. 2014). In the

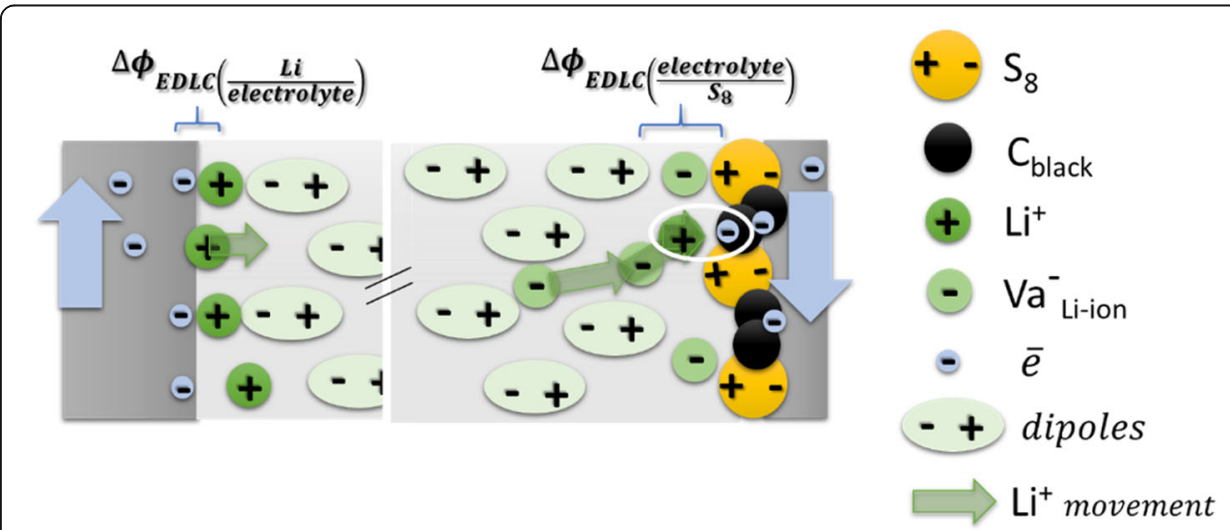

Fig. 5 Schematic of EDLCS at the electrode and electrolyte interfaces during discharge where electric dipoles coexist with the mobile cations and negatively charged vacancies in the electrolyte. The positive electrode's incoming electrons tunnel through the quantum triangular barrier formed by the EDLC that equalizes its electrochemical potential of the electrolyte with the electrochemical potential of the positive electrode; each tunneling electron reduces a $\mathrm{Li}^{+}$into $\mathrm{Li}^{0}$. The dipoles help to keep the ions confined at the interfaces 


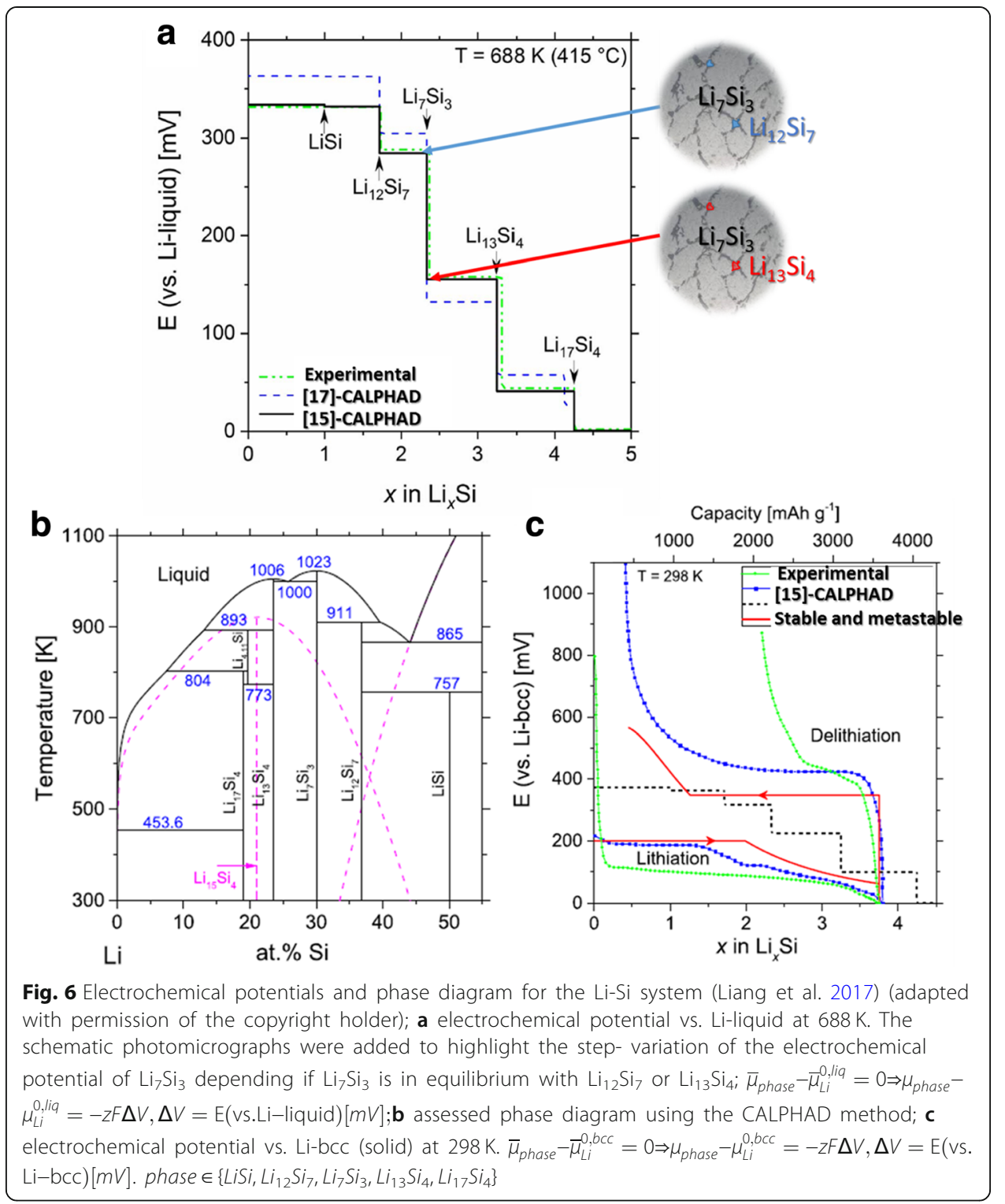

phase $\mathrm{Li}_{7} \mathrm{Si}_{3}$, the electrochemical potential varies from -15 to $-28 \mathrm{~kJ} \cdot \mathrm{mol}_{\text {of atoms }}{ }^{-1}$ (at $688 \mathrm{~K})\left(\right.$ Fig. $7 \mathrm{c}$ ). The variation of the electrochemical potential of the lithium in $\mathrm{Li}_{7} \mathrm{Si}_{3}$ it is not due to a stoichiometric change; it happens because $\mathrm{Li}_{7} \mathrm{Si}_{3}$ is either in contact with $\mathrm{Li}_{13} \mathrm{Si}_{4}$ at $\mu_{L i}-\mu_{L i}^{0, L i q}=-15 \mathrm{~kJ} \cdot \mathrm{mol}_{\text {of atoms }}{ }^{-1}$ or with $\mathrm{Li}_{12} \mathrm{Si}_{7}$ at $\mu_{L i}-\mu_{L i}^{0, L i q}=-28$ $\mathrm{kJ} \cdot \mathrm{mol}_{\text {of atoms }}{ }^{-1}$ as shown before for Fig. 6. In other words, a small or big amount of $\mathrm{Li}_{7} \mathrm{Si}_{3}$ (depending on the lever rule) in equilibrium with $\mathrm{Li}_{12} \mathrm{Si}_{7}$ shows the same electrochemical potential as $\mathrm{Li}_{12} \mathrm{Si}_{7}$; similarly, $\mathrm{Li}_{7} \mathrm{Si}_{3}$ in equilibrium with $\mathrm{Li}_{13} \mathrm{Si}_{4}$ shows the same electrochemical potential as $\mathrm{Li}_{13} \mathrm{Si}_{4}$. The same happens for all other phases as shown in Figs. 6 and 7, including $\mathrm{Li}$ and $\mathrm{Si}$ solid solutions, and all the phase diagrams.

Therefore, in our cell and in our positive electrode, $\mathrm{Li}$ in contact with the bulk electrode will show the same Fermi level as the positive electrode provided it does not cover a critical amount of the surface in contact with the electrolyte. The chemical 


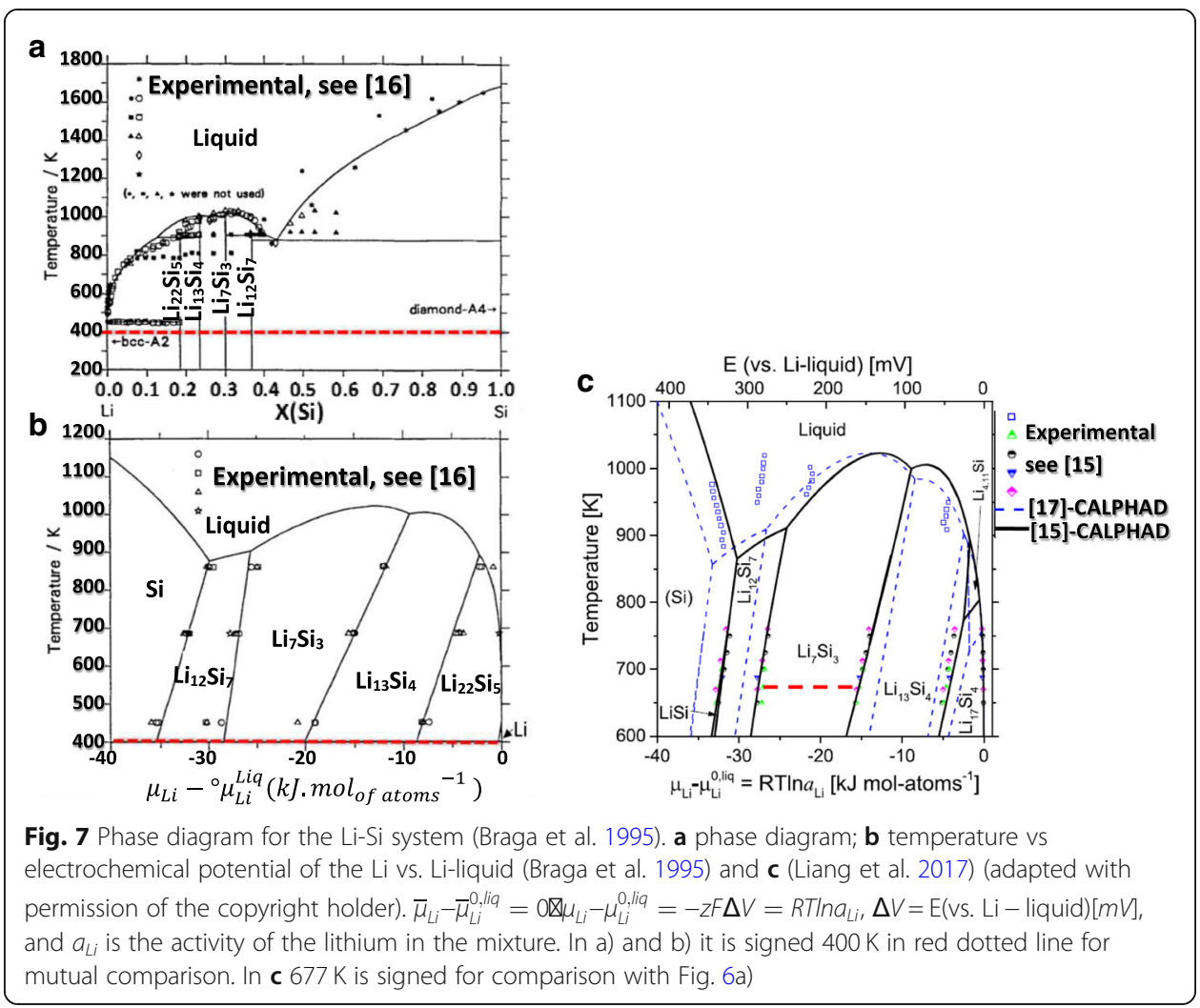

potential of the positive electrode is constrained by the electrochemical potential of the insulator because of the previously described reasons.

\section{Experimental}

For the $\mathrm{SS}+\mathrm{Li} / \mathrm{Li}^{+}$-glass $/ \mathrm{S}+\mathrm{C}+\mathrm{Al}+\mathrm{SS}$ cell, a positive electrode slurry containing 60 wt\% of $\mathrm{S}_{8}(1.08 \mathrm{mg}), 30 \mathrm{wt} \%(0.54 \mathrm{mg})$ of carbon black Super P and $10 \mathrm{wt} \%$ PVDF $(0.18 \mathrm{mg})$ was prepared as in (Braga et al. 2018). The slurry was then doctor bladed onto an aluminum foil current collector. The Li-glass electrolyte in a non-woven recyclable paper matrix was prepared as in (Braga et al. 2017; Braga et al. 2018). The cell was assembled in a CR2032 casing. The Li content was $3.1 \mathrm{mg}$.

A LANHE battery tester was used to discharge the cell. The instrument is different from the one used in (Braga et al. 2017).

After discharge, the cell was opened inside an argon-filled glove box by removing the loosely bound positive electrode Al-current collector. The electrode and a piece of scratched lithium metal for reference were transferred into an ultrahigh-vacuum X-ray photoelectron-spectrometer chamber without exposing them to air by employing a sample transfer interface. An XPS study was performed with a Kratos Axis Ultra, a multitechnique electron spectrometer (Manchester, UK). The monochromatic Al X-ray source $\left(148.6 \mathrm{eV}\right.$ ) was calibrated with the $3 \mathrm{~d}_{5 / 2}$ line of silver (Hoffmann et al. 2015). Casa XPS analysis software was used for peak deconvolution, and line syntheses were conducted with a Gaussian-Lorentzian 70:30 curve fit and linear background subtraction of elemental spectra. The XPS profile depth was $10 \mathrm{~nm}$ (Hoffmann et al. 2015; Singh et al. 2015). 

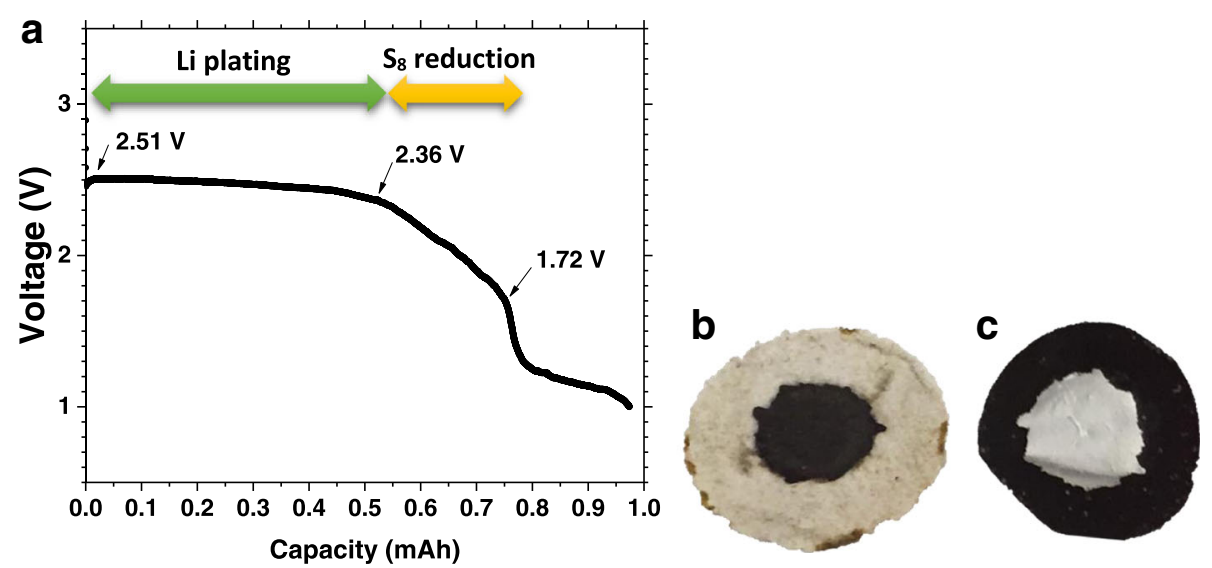

Fig. 8 Primary SS + Li/Li-glass/S $+\mathrm{C}+\mathrm{Al}+\mathrm{SS}$ cell. a discharge cycle. $\mathrm{S}_{8}$ is not reduced while the cell voltage varies from 2.51 to $2.36 \mathrm{~V}$; in this voltage range, Li was plated. From 2.36 to $1.3 \mathrm{~V}$, $\mathrm{S}_{8}$ reduction occurs. The $\mathrm{S}_{8}$ reduced corresponded to around $1 / 10$ of the $\mathrm{S}_{8}$ in the positive electrode. $\mathbf{b}$ Photograph of the positive electrode on the paper matrix containing the Li-glass electrolyte; lithium of the negative electrode (not shown) is on the opposite side of the glass-on-paper. c The removed Al-positive electrode current collector

\section{Results and discussion}

Figure 8a shows the discharge voltage of an asymmetric $\mathrm{SS}+\mathrm{Li} / \mathrm{Li}^{+}$-glass $/ \mathrm{S}+\mathrm{C}+\mathrm{Al}+$ SS cell in which the carbon of the positive electrode delivers the electrons from the negative electrode that plate the $\mathrm{Li}^{+}$from the electrolyte at a voltage determined by the chemical potential of the sulfur $\mathrm{S}_{8}$ (from 2.51 to $2.36 \mathrm{~V}$ ). Below $2.36 \mathrm{~V}$, the sulfur starts to be reduced. The $S_{8}$ reduced below $2.36 \mathrm{~V}$ corresponded to around $1 / 10$ of the $S_{8}$ in the positive electrode (considering the theoretical capacity of $\mathrm{S}_{8}, 1675 \mathrm{~mA} \cdot \mathrm{g}^{-1}$ ) which is acceptable since not all of the thick positive electrode was in contact with the electrolyte, as is shown in Fig. 8b-c.

In Fig. 8, the profile of $\mathrm{V}_{\text {dis }}$ versus capacity, which is proportional to time for a constant discharge current with an abrupt change at $\mathrm{V}_{\text {dis }}=2.36 \mathrm{~V}$ in the rate of reduction of $\mathrm{V}_{\text {dis }}$ provides a quantitative agreement with prediction for a cell with lithium negative-electrode and a positive-electrode electrochemical potential set by the $S_{8}$ relay (Fig. 4). The rate of reduction of $\mathrm{V}_{\text {dis }}$ increases abruptly once a large number of $\mathrm{S}_{8}$ particles have their surface states completely occupied (Fig. 3).

Figure 9 shows XPS data for the positive electrode and lithium-metal reference; they provide direct evidence of lithium plated on the positive electrode. Recent literature (Hoffmann et al. 2015) shows that a potential is created on the surface of the sample that creates a shift in peak positions when taking spectra of lithium metal, thus causing the adjustment of the carbon spectra to account for the presence of adventitious carbon to be non-trivial.

If the adventitious carbon peaks in the spectra were to be taken as reference, as is traditionally done in the literature, the Li-metal peaks would be matching the Li-metal, as observed in Fig. 9b.

\section{Examples from the literature}

In a recent paper, F. Leng et al. (Lenga et al. 2017) have studied the degradation processes in lithium-ion commercial batteries during aging. Graphite/lithium cobalt oxide 


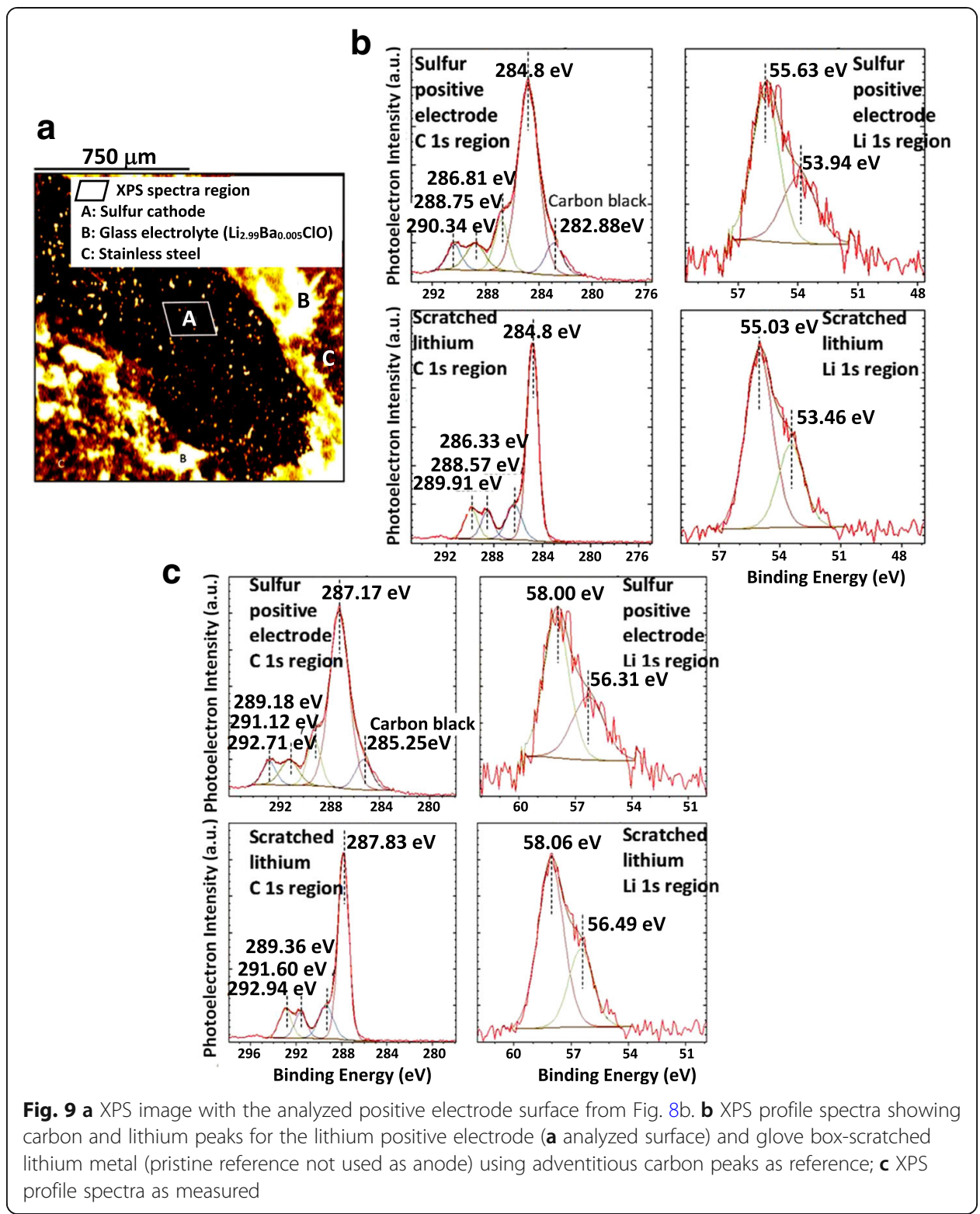

(LCO) coin cells were subjected to up to 1000 galvanostatic charge-discharge cycles. Experiments show that between 600 and 800 cycles, lithium plated in the positive electrode. According to (Lenga et al. 2017), between 600 and 800 cycles, a previously formed LCO surface-insulating layer will crack, resulting in surface spallation with subsequent clogging of the separator's pores. It is pointed out that at this point, plating conditions are created at the positive electrode and an insulating layer relay in contact with the electrolyte fixes the electrochemical potential of the positive electrode, not allowing the LCO to be immediately reduced upon discharge and facilitating lithium plating.

In an in-situ neutron diffraction study of commercial jelly-roll NMC/graphite battery cell at sub-ambient temperatures, V. Zinth et al. (Zinth et al. 2014) did not identify plating on the positive electrode while discharging at $C / 10$ after charging at $C / 5$, at $-20{ }^{\circ} \mathrm{C}$. A careful analysis of the experiments shows that indeed lithium was plated 


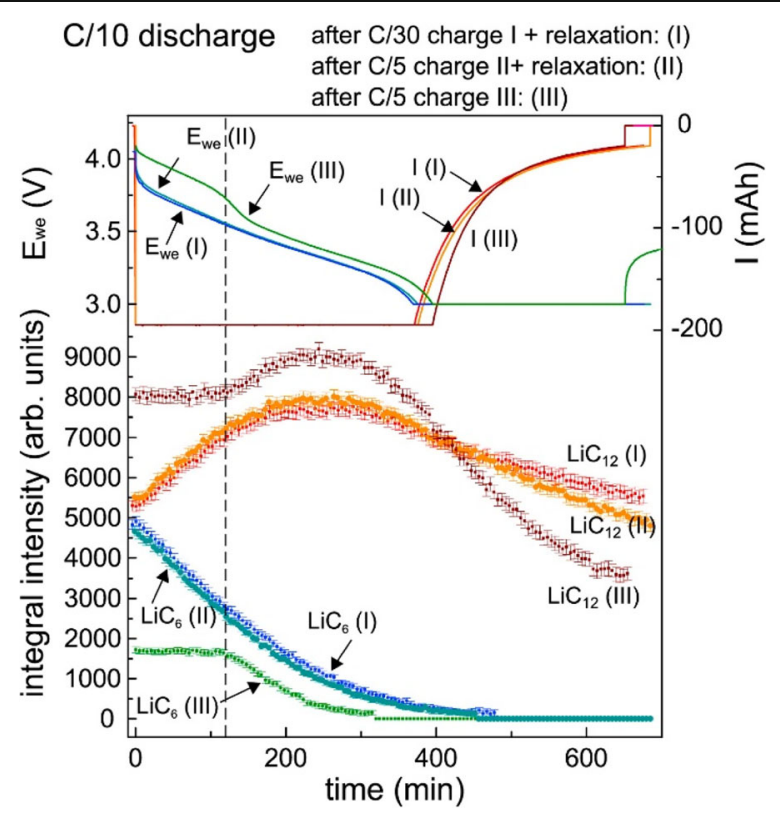

Fig. 10 Integral reflection intensities of $\mathrm{LiC}_{12}$ and $\mathrm{LiC}_{6}$ during $\mathrm{C} / 10$ discharge after $\mathrm{C} / 30$ charge (red/blue), $\mathrm{C} / 5$ charge + rest period (orange/cyan) and $\mathrm{C} / 5$ charge without rest period (green/pink). Voltage (working potential Ewe) and current during discharge (top) (Zinth et al. 2014) (reproduced with permission of the copyright holder)

on the positive electrode during discharge. A plateau starting at $4.1 \mathrm{~V}$ that was not correlated with either lithium plating on the surface of the negative electrode (related with the $\mathrm{LiC}_{12}$ peak) or with $\mathrm{LiC}_{6}$ concentrations change, indicates that lithium was being plated on the positive electrode from the electrolyte for around $120 \mathrm{~min}$. Increasing the capacity of the cell (Fig. 10). After $120 \mathrm{~min}$., $\mathrm{LiC}_{6}$ starts to decrease while $\mathrm{LiC}_{12}$ increases, showing the oxidation process of the negative electrode. It is highlighted that the low temperature helps to prevent reduction of the NMC.

\section{Conclusions}

The observation of the transfer of metallic-lithium from the negative electrode to the positive electrode on which an insulator relay on the positive electrode contacts the electrolyte is shown theoretically and experimentally to conform to the laws of Thermodynamics. The concept of transfer of an alkali-metal between the two electrodes at a finite voltage is not restricted to a $\mathrm{Li}^{+}$electrolyte and to a sulfur relay; it is also applicable to $\mathrm{Na}^{+}$and $\mathrm{K}^{+}$electrolytes and other semiconductors and insulator relays.

\section{Additional file}

Additional file 1: Supplementary Information Thermodynamic considerations of same-metal electrodes in an asymmetric cell. (DOC $263 \mathrm{~kb}$ )

\section{Abbreviations}

CALPHAD: Calculation of Phase Diagrams; DFT: Density Function Theory; EDLC: Electrical Double Layer Capacitor; LCO: Lithium cobalt oxide; LED: Light emitting diode; LHB: Lower Hubbard band; PVDF: Polyvinylidene fluoride;

SS: Stainless steel; UHB: Upper Hubbard band; XPS: X-ray Photoelectron Spectroscopy

Funding

MHB acknowledges the COMPETE2020 and FCT project, EU and Portugal, PTDC/CTM-ENE/2391/2014. 
Availability of data and materials

Data sharing not applicable to this article as no datasets were generated or analyzed during the current study besides the data that is shown in the article.

\section{Authors' contributions}

MHB conceived of the study, experiments, analysis, theoretical arguments, ab initio and tunneling calculations and wrote the manuscript with JBG. NSG prepared battery cells, test them and performed XPS. AJM synthesized the electrolyte and prepared battery cells. JBG discussed theoretical arguments and wrote the manuscript. All authors read and approved the final manuscript.

Competing interests

The authors declare that they have no competing interests.

\section{Publisher's Note}

Springer Nature remains neutral with regard to jurisdictional claims in published maps and institutional affiliations.

\section{Author details}

${ }^{1}$ Texas Materials Institute and the Materials Science and Engineering Program, The University of Texas at Austin, Austin TX, USA. 'LAETA, Engineering Physics Department, Engineering Faculty, University of Porto, R. Dr. Roberto Frias $s / \mathrm{n}$, 4200-465 Porto, Portugal.

Received: 8 June 2018 Accepted: 14 December 2018

Published online: 25 January 2019

\section{References}

J. Bardeen, Phys. Review 71(10), 717 (1947) and H. Kroemer, Nobel Lecture, Dec. 8, 2000, https://www.nobelprize.org/uploads/ 2018/06/kroemer-lecture.pdf

M.H. Braga, A. Debski, W. Gasior, J. Alloys Compd., 616, 581-593 (2014)

M.H. Braga, N.S. Grundish, A.J. Murchison, J.B. Goodenough, Energy Environ. Sci. 10, 331-336 (2017)

M.H. Braga, L.F. Malheiros, I. Ansara, J. Phase Equilibria 16(4), 324-330 (1995)

M.H. Braga, C.M. Subramaniyam, A.J. Murchison, J.B. Goodenough, J. Am, Chem. Soc. 140(20), 6343-6352 (2018)

E.N. Brothers, A.F. Izmaylov, J.O. Normand, V. Barone, G.E. Scuseria, J. Chem. Phys. 129, 011102 (2008)

S. Heo, J. Kim, G.K. Ong, D.J. Milliron, Nano Lett. 17(9), 5756-5761 (2017)

M. Hoffmann, M. Zier, S. Oswald, J. Eckert, J. Power Sources 288, 434-440 (2015)

J. Jenkins, W.H. Jarvis, Thermionics: Basic principles of electronics, vol 1 (Elsevier, 2013)

C.J. Kaiser, The capacitor handbook: a comprehensive guide for correct component selection in all circuit applications. Know what to use when and where, 2nd edn. (C J Publishing, 2011)

C. Kittel, H. Kroemer, Thermal physics, 2nd edn. (W. H. Freeman, 1980)

F. Lenga, Z. Wei, C.M. Tan, R. Yazami, Electrochim. Acta 256, 52-62 (2017)

S.-M. Liang, F. Taubert, A. Kozlov, J. Seidel, F. Mertens, R. Schmid-Fetzer, Intermetallics 81, 32-46 (2017)

M. Nonnenmacher, M.P. O'Boyle, H.K. Wickramasinghe, Appl. Phys. Lett. 58(25), 2921-2923 (1991)

NREL, Basic photovoltaic principles and methods, SERI/SP-290-1448, 1982

I. Prigogine, R. Defay, Chemical thermodynamics (Longmans, Green \& Co, London, 1950/1954)

J.B. Rivest, G. Li, I.D. Sharp, J.B. Neaton, D.J. Milliron, J. Phys. Chem. Lett. 5(14), 2450-2454 (2014)

E.F. Schubert, Light-emitting diodes, 2nd edn. (Cambridge University Press, 2006)

P. Singh, K. Shiva, H. Celio, J.B. Goodenough, Energy Environ. Sci. 8, 3000-3005 (2015)

J. Sparkes, Semiconductor devices, 2nd edn. (CRC Press, 1994)

S. Trasatti, Pure Appl. Chem. 58(7), 955-966 (1986)

V. Zinth, C. von Lüders, M. Hofmann, J. Hattendorff, I. Buchberger, S. Erhard, J. Rebelo-Kornmeier, A. Jossen, R. Gilles, J. Power Sources 271, 152-159 (2014)

\section{Submit your manuscript to a SpringerOpen ${ }^{\circ}$ journal and benefit from:}

- Convenient online submission

- Rigorous peer review

- Open access: articles freely available online

High visibility within the field

- Retaining the copyright to your article

Submit your next manuscript at $\boldsymbol{\nabla}$ springeropen.com 